\title{
Light charged particles emitted in fission reactions induced by protons on ${ }^{208} \mathrm{~Pb}$
}

J. L. Rodríguez-Sánchez, ${ }^{1}$ J. Benlliure, ${ }^{1}$ C. Paradela, ${ }^{1,}{ }^{*}$ Y. Ayyad,${ }^{1, \dagger}$ E. Casarejos,${ }^{2}$ H. Alvarez-Pol,${ }^{1}$ L. Audouin, ${ }^{3}$ G. Bélier, ${ }^{4}$ G. Boutoux ${ }^{4,}$ A. Chatillon, ${ }^{4}$ D. Cortina-Gil,${ }^{1}$ T. Gorbinet, ${ }^{4}$ A. Heinz,${ }^{5}$ A. Kelić-Heil, ${ }^{6}$ B. Laurent, ${ }^{4}$ J.-F. Martin, ${ }^{4}$ E. Pellereau, ${ }^{4}$ B. Pietras, ${ }^{1}$ D. Ramos, ${ }^{1}$ C. Rodríguez-Tajes, ${ }^{1,7}$ D. M. Rossi, ${ }^{6}$ H. Simon, ${ }^{6}$ J. Taïeb, ${ }^{4}$ J. Vargas, ${ }^{1}$ and B. Voss ${ }^{6}$

${ }^{1}$ Universidad de Santiago de Compostela, E-15782 Santiago de Compostela, Spain

${ }^{2}$ Universidad de Vigo, E-36200 Vigo, Spain

${ }^{3}$ Institut de Physique Nucléaire d'Orsay, F-91406 Orsay, France

${ }^{4}$ CEA, DAM, DIF, F-91297 Arpajon, France

${ }^{5}$ Chalmers University of Technology, SE-412 96 Gothenburg, Sweden

${ }^{6}$ GSI-Helmholtzzentrum für Schwerionenforschung GmbH, D-64291 Darmstadt, Germany

${ }^{7}$ Grand Accélérateur National d'Ions Lourds, F-14076 Caen Cedex 05, France

(Received 18 May 2016; published 8 September 2016)

\begin{abstract}
Light charged particles emitted in proton-induced fission reactions on ${ }^{208} \mathrm{~Pb}$ have been measured at different kinetic energies: $370 \mathrm{~A}, 500 \mathrm{~A}$, and $650 \mathrm{~A} \mathrm{MeV}$. The experiment was performed by the SOFIA Collaboration at the GSI facilities in Darmstadt (Germany). The inverse kinematics technique was combined with a setup especially designed to measure light charged particles in coincidence with fission fragments. This measurement allowed us, for the first time, to obtain correlations between the light charged particles emitted during the fission process and the charge distributions of the fission fragments. These correlations were compared with different model calculations to assess the ground-to-saddle dynamics. The results confirm that transient and dissipative effects are required for an accurate description of the fission observables.
\end{abstract}

DOI: 10.1103/PhysRevC.94.034605

\section{INTRODUCTION}

In recent decades, many experimental and theoretical studies on dissipative effects in fission have been performed $[1,2]$. The dissipation or friction force enlarges the fission time with respect to statistical times calculated according to the transition-state model [3]. Dissipative effects also reduce the fission probability because of the lower fission decay widths, as pointed out by Kramers in 1940 [4], but also because the longer fission time favors the deexcitation of the compound nuclei by other competing processes, such as particle evaporation.

The study of the role of dissipative effects in fission relies on transport equations, such as the Langevin or FokkerPlanck equations. The latter was used by Grangé, Bhatt, and collaborators $[5,6]$ to show that nuclear dissipation also affects the fission process by increasing the ground-to-saddle time, leading to the so-called transient time.

This transient time or fission delay affects many experimental observables, such as the total and partial fission cross sections [7-15], the charge and mass distributions of the fission fragments [10,11,15-19], the neutron multiplicities [1,20-23], and the multiplicity of prescission light charged particles [24] and $\gamma$ rays [25,26]. The observation of an excess of prescission neutron [21-23,27] and $\gamma$-ray [21-23,25] multiplicities with respect to the predictions of the statistical model, for nuclei in a wide range of fissility, has been the most direct confirmation of dynamical effects in fission. This conclusion was also

\footnotetext{
${ }^{*}$ Present address: EC-JRC, Institute for Reference Materials and Measurements, Retieseweg 111, B-2440 Geel, Belgium.

${ }^{\dagger}$ Present address: National Superconducting Cyclotron Laboratory, Michigan State University, East Lansing, MI 48824-1321, USA.

${ }^{\ddagger}$ Present address: Université Bordeaux, F-33405 Talence, France.
}

supported by the observation of a reduction of the fission cross sections $[9,12-14]$ and the widths of the charge distributions of the fission fragments $[10,15,16,19]$ with respect to the statistical model.

However, different attempts to constrain the magnitude of the dissipation parameter used to describe the fission dynamics with transport equations yield quite different results [1,2], leading to transient times between $1 \times 10^{-21}$ and $30 \times$ $10^{-21}$ s $[7,8,14,19,24,28,29]$. Moreover, the dependence of the dissipation parameter on the nuclear shape and temperature is still largely debated $[2,21-23,26,27]$. This fact is most likely caused by the lack of constrains for model calculations, which are crucial given their dependence on many parameters, such as the dissipation coefficient, level densities, and fission barriers. Consequently, the comparison of several independent observables measured in coincidence with the predictions obtained with model calculations could improve the situation.

Measurements of light particles have been used as a "clock" to estimate the fission time [24,30,31] because they are emitted along the fission path up to the scission-point configuration. In particular, Lestone et al. [32] used fusion-fission reactions to investigate the pre- and post-saddle fission times by comparing proton- and $\alpha$-particle multiplicities with statistical model calculations. Their work demonstrated that this observable is sensitive to dissipation. Similar conclusions were obtained by Fröbrich and Gontchar [33] by comparing the same data with dynamical calculations based on the Langevin equation.

On the other hand, some recent works, based on the measurement of light-particle multiplicities produced in spallation reactions at high excitation energies in direct kinematics [34-39], have pointed out that these dissipative and transient time effects underestimate the fission probability at high excitation energies. In these experiments, model calculations 
were used to correlate the excitation energy of the compound systems at the ground state with particle multiplicity [34]. However, the correlation between the excitation energy and the multiplicity of light particles could change with the model parameters, as demonstrated in Refs. [29,30,40-42], affecting the conclusions.

To go further in the investigation of fission dynamics, a novel experimental setup designed by the SOFIA (studies on fission with ALADIN) Collaboration [43-49] was used to measure many of these observables in coincidence. In our previous publications [28,50,51] we used total and partial fission cross sections and the distributions in atomic number of the final fission fragments to investigate ground-to-saddle dissipative effects in fission. These works demonstrated that a constant value of the dissipation parameter can describe a large number of fission observables. Furthermore, the results revealed that the dissipation coefficient is independent of temperature [51].

In those works we took advantage of the high excitation energies and low angular momenta and shape distortion induced by spallation reactions. However, these initial conditions for the fissioning nuclei could only be estimated with model calculations. In the present work we propose to use the multiplicity and atomic number of the light charged particles emitted in fission reactions to constrain the initial excitation energy. In this kind of reaction, light charged particles can be emitted during the first stage of the reaction, the so-called intranuclear cascade or abrasion process, or in the deexcitation of the compound nucleus. In the latter case we can assume that light charged particles are mostly emitted before the saddle point because of the shorter saddle-to-scission paths, where the most probable deexcitation channels are the emission of neutrons and $\gamma$ rays. The measurement of these particles in coincidence with fission is a difficult task in direct kinematics experiments due to the fact that fission fragments cannot always be detected, and it is even harder to identify them in terms of their atomic or mass number [34,36]. For this reason, our novel experimental setup together with the inverse kinematics technique represent an ideal scenario because fission fragments and light charged particles leave the target with high kinetic energies in the forward direction, facilitating their detection with high efficiency and precision.

\section{EXPERIMENT}

The experiment was carried out at the GSI facilities in Darmstadt (Germany), where the SIS18 synchrotron was used to accelerate ions of ${ }^{208} \mathrm{~Pb}$ at $370 A, 500 A$, and $650 A \mathrm{MeV}$ with an intensity around $10^{5}$ ions/s. Fission reactions were produced in a cylindrical target filled with liquid hydrogen $\left(\sim 85 \mathrm{mg} / \mathrm{cm}^{2}\right)$. In addition, we also measured the reaction ${ }^{208} \mathrm{~Pb}(500 \mathrm{~A} \mathrm{MeV})+{ }^{27} \mathrm{Al}$.

The inverse kinematics technique used in this work offers several advantages in measuring the light charged particles with respect to conventional experiments performed in direct kinematics because it provides an accurate and unambiguous identification of fission products. In this case, the fission fragments and light charged particles emitted in the deexcitation process leave the target in the forward direction with high
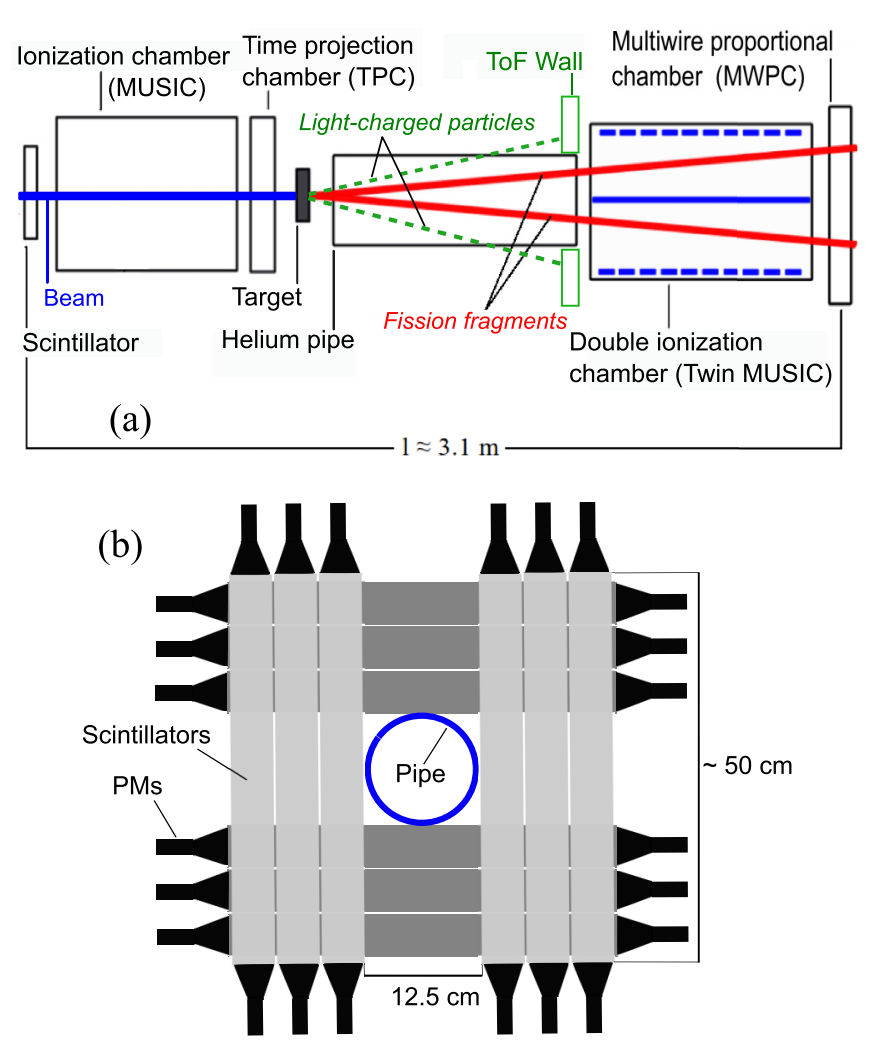

FIG. 1. (a) Schematic diagram of the experimental setup used to measure the light charged particles in coincidence with fission. Sizes are not to scale. (b) Schematic view of the ToF wall used to detect the light charged particles.

kinetic energies, covering narrow angular ranges up to 50 and $250 \mathrm{mrad}$, respectively.

\section{A. Experimental setup}

In this experiment we took advantage of the SOFIA detection setup [43-49]. The most relevant features of this setup for the present measurement are described in the following.

A multisampling ionization chamber (MUSIC) and a time projection chamber (TPC) were placed upstream of the target providing the beam identification and its position on the liquid-hydrogen cell, as shown in Fig. 1(a). Then, a double multisampling ionization chamber (twin MUSIC) [52], placed downstream of the target, was used to detect both fission fragments simultaneously and permitted us an unambiguous identification of fission [28]. In addition, the tracking capabilities of the twin MUSIC allowed us to select fission events produced at the target position [28].

Light-charged particles were detected in a time-of-flight (ToF) wall placed behind the target at a distance of $140 \mathrm{~cm}$. This detector, depicted in Fig. 1(b), consisted of two detection planes made of segmented plastic scintillators, each $50 \mathrm{~cm}$ long, $6 \mathrm{~cm}$ wide, and $1 \mathrm{~cm}$ thick. The first plane was formed by six horizontal paddles and the second one by six vertical paddles, which left a square hole of $12.5 \times 12.5 \mathrm{~cm}^{2}$ in the center for the transmission of the fission fragments through a pipe 
filled with helium gas, as shown in Fig. 1(b). The scintillators were coupled to Hamamatsu R8619 photomultipliers (PMs) in both ends to process their signals. The time and charge of the scintillator signals were registered using a time-to-digital converter (TDC) and a charge-to-digital converter (QDC), respectively.

\section{B. Identification of light charged particles and detection efficiency}

Because of the kinematics, while the fission fragments are emitted with polar angles up to $50 \mathrm{mrad}$, covered by the twin MUSIC [28], a major fraction of the light charged particles are emitted at larger polar angles covered by the ToF wall. The identification matrix of light- charged particles with this ToF wall is displayed in Fig. 2(a). This figure shows a scatter plot of the time of flight of the detected light charged particles emitted in fission events as a function of their energy loss in the plastic scintillators. As can be seen, with these measurements one can identify particles with atomic numbers between $Z=1$ and $Z=3$. Heavier particles are also produced but cannot be unambiguously identified with the achieved resolution. In the inset of the figure, we also represent the charge distribution of the detected particles.

The detection of light charged particles is affected by the geometrical acceptance of our detection setup and by the intrinsic efficiency of the plastic scintillators. To determine the intrinsic efficiency for the detection of $Z=1$ particles we took advantage of fission events producing fragments such that $Z_{1}+Z_{2}=81$. The ratio between the number of detected protons and those fission events provides the total efficiency for the detection of $Z=1$ particles. Correcting this efficiency by the geometrical acceptance obtained from GEANT4 simulations [53] we can determine the intrinsic efficiency for the detection of $Z=1$ particles that amounts to $(85 \pm 3) \%,(80 \pm 3) \%$, and $(75 \pm 3) \%$ for the projectile kinetic energies of $370 \mathrm{~A}, 500 \mathrm{~A}$, and $650 \mathrm{~A} \mathrm{MeV}$, respectively. In this case, we also assume that the detection of neutrons due to elastic or inelastic reactions is negligible because the probability for such reactions is below $0.2 \%$. For light charged particles with $Z>1$ the intrinsic efficiency was assumed to be around $100 \%$.

These intrinsic efficiencies were then included in the simulations to obtain realistic total correction factors for larger multiplicities of light charged particles with $Z=1$. For these simulations we used as event generator the codes INCL4.6 [54] and ABLA07 [55], which provide a satisfactory description of the kinematics of the particles [54]. We also took the dimensions and positions of the detection paddles of the ToF wall into account.

The total correction factors for $Z=1$ particles, representing a convolution of the intrinsic efficiency with the geometrical acceptance, are displayed in Fig. 2(b) as a function of their multiplicity, together with their associated uncertainties (dashed area). According to these results, the larger multiplicities contaminate the lower ones and they need to be corrected by factors larger than 1 .

In Fig. 3 we show the measured yields (solid triangles) of particles with $Z=1$ and the same yields after applying the total correction factors (open squares). Finally, due to the fact
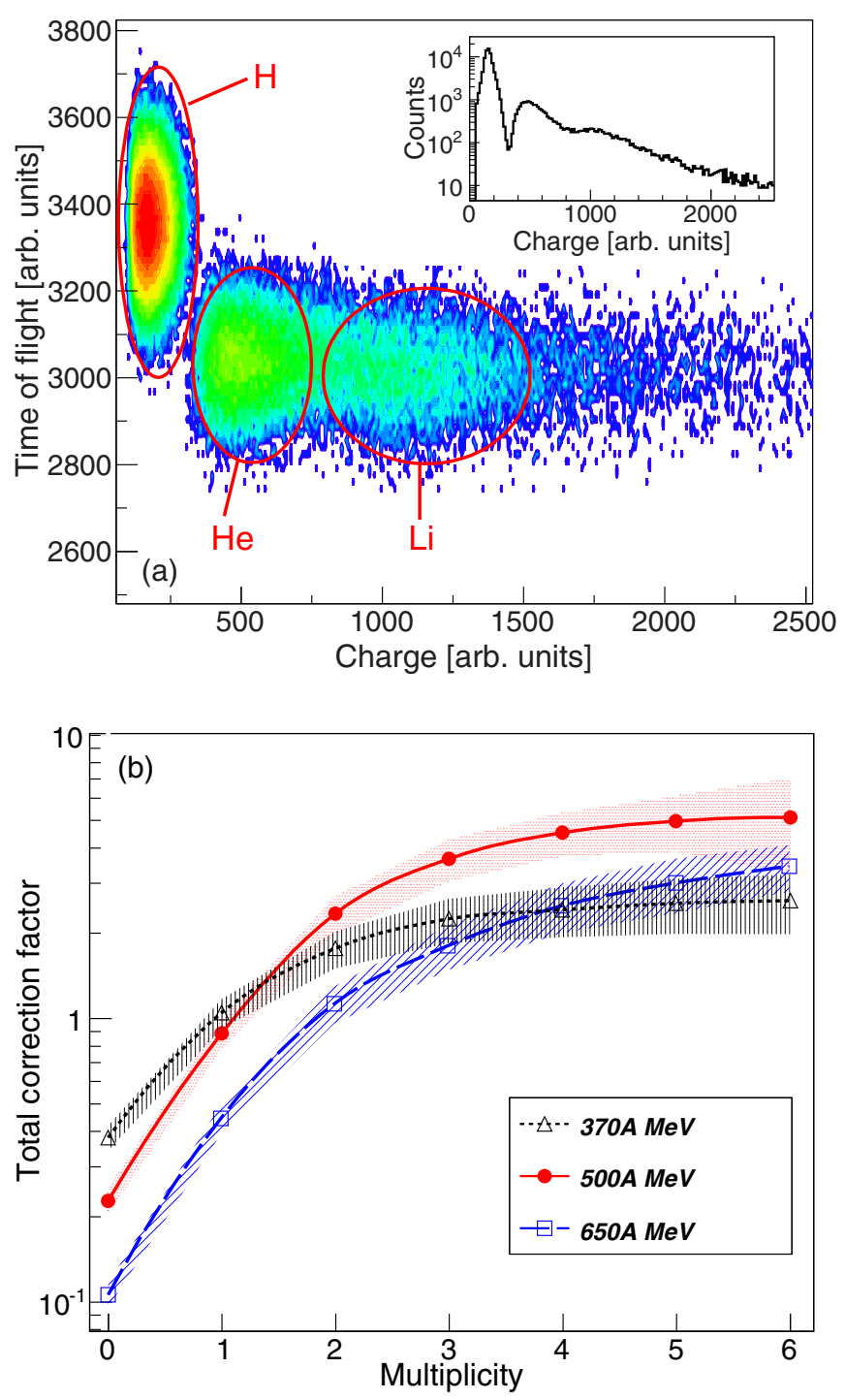

FIG. 2. (a) Identification of light charged particles emitted in fission in the scintillators of the ToF wall. The solid ellipses indicate the different particles. The inset shows the projection of the charge distribution. (b) Total correction factor for light charged particles with $Z=1$ as a function of its multiplicity. These correction factors are calculated for the three kinetic energies investigated in this work. The uncertainties are shown with dashed areas.

that the maximum multiplicity detected by the ToF wall is six (maximum number of paddles), the tails of the corrected yields (open squares) were extrapolated with a Gaussian fit (solid line) to determine the contribution of the largest multiplicities.

\section{RESULTS AND DISCUSSION}

Light charged particle multiplicity is one of the most widely used observables to investigate the dynamics of the fission process in direct kinematics [31,56-58]. However, because of the required technological developments, no data were taken in inverse kinematics prior to this work. In this sense, our experimental setup provides the first measurement of the light charged particle multiplicity in coincidence with 


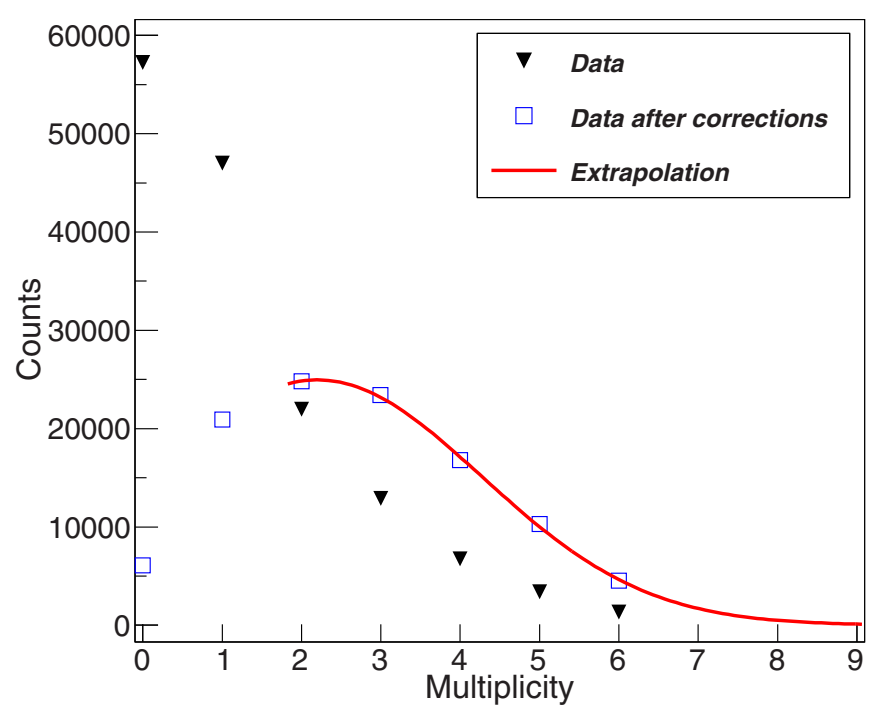

FIG. 3. The measured distribution of particles with $Z=1$ for the reaction ${ }^{208} \mathrm{~Pb}+p$ at $650 \mathrm{~A} \mathrm{MeV}$ is shown in comparison to the one after corrections. The uncertainties are shown if they exceed the size of the symbols. The solid line represents a Gaussian extrapolation.

an unambiguous identification of the fission fragments. In our previous works [28,51] we used the identification of the fission fragments to investigate the role of the level-density and the dissipation parameters in the description of fission, by comparing the total and partial fission cross sections and the widths of the atomic-number distribution of the fission fragments with different model calculations. In the present work we use the average multiplicity of particles identified with atomic number $Z=1$ to confirm the results about the role of dissipation in fission. Moreover, the correlations between the charge identification of the emitted light charged particles and the fission fragments allow us to add more constraints to the initial excitation energy of the fissioning systems.

Figure 4(a) shows the probability for the emission of particles with $Z=1$ in fission events as function of the total multiplicity of light charged particles per event for the reaction ${ }^{208} \mathrm{~Pb}+p$ at $370 \mathrm{~A}$ (open triangles), $500 \mathrm{~A}$ (solid circles), and $650 A$ (open squares) MeV. These distributions were obtained after applying the correction factors shown in Fig. 2(b) to the measured multiplicity distributions.

In Fig. 4(a) the maximum of these distributions appears for small values of the total multiplicity, as expected because most of the fissioning systems are produced in peripheral collisions where only a few of nucleons are removed. Then, the tails observed towards larger multiplicities can be attributed to the production of lighter fissioning nuclei with higher excitation energies [15,51]. As can also be seen in the figure, the probability for fission events with large multiplicities of light charged particles emitted in coincidence, as well as the maximum of the distributions, increase with the bombarding energy. This tendency can be attributed to the increase of the violence of the reaction, which means more particles are emitted during the intranuclear cascade and deexcitation processes.
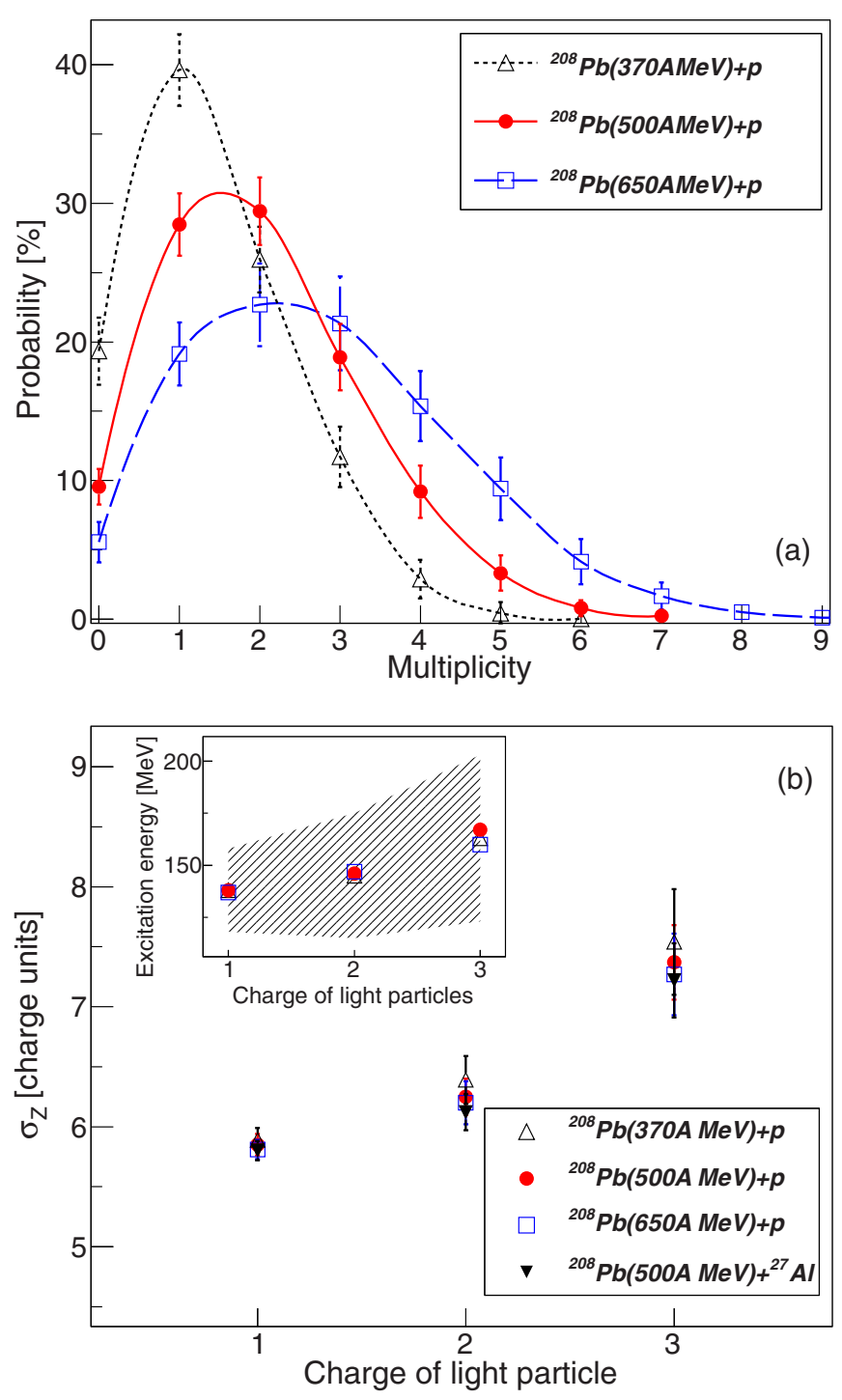

FIG. 4. (a) Multiplicity distribution of particles with $Z=1$ for the reaction ${ }^{208} \mathrm{~Pb}+p$ at different kinetic energies: $370 A$ (open triangles), $500 \mathrm{~A}$ (solid circles), and 650A (open squares) $\mathrm{MeV}$. The lines are to guide the eye. (b) Width of the atomic-number distribution of the final fission fragments as a function of the atomic number of the removed light charged particle from the projectile. The data for the reaction ${ }^{208} \mathrm{~Pb}+{ }^{27} \mathrm{Al}$ at $500 \mathrm{~A} \mathrm{MeV}$ (solid triangles) are also displayed. The inset shows the excitation energy as a function of the size of the emitted particle, where the dashed area indicates the width of the distribution.

From previous works $[10,15,16,19,51,59]$, we know that the width of the atomic-number distribution of the fission fragments is strongly correlated with the excitation energy of the compound nuclei. In particular, this observable should be proportional to the temperature at the saddle-point deformation according to a statistical interpretation [60] [see Eq. (1)]. The investigation of this observable as a function of the atomic number of the emitted light charged particle could allow us to constrain its evolution with the initial excitation energy, because most of the light charged particles are emitted during the intranuclear cascade process [54]. In this sense, we propose 
to study the width of the atomic-number distribution of the fission fragments as a function of the atomic number of the light charged particle emitted in coincidence with fission, as shown in Fig. 4(b). Moreover, restrictions in the atomic number of the fissioning system $\left(Z_{1}+Z_{2}\right)$ were also considered to avoid contaminations of higher proton multiplicities: $Z_{1}+Z_{2}>80$ for $Z=1, Z_{1}+Z_{2}>79$ for $Z=2$, and $Z_{1}+Z_{2}>78$ for $Z=3$. These conditions also guarantee that the light charged particles originate only from the intranuclear cascade process. This observable is displayed for different reactions: ${ }^{208} \mathrm{~Pb}+p$ at $370 \mathrm{~A}$ (open triangles), $500 \mathrm{~A}$ (solid circles), and 650 $\mathrm{A}$ (open squares) $\mathrm{MeV}$ and ${ }^{208} \mathrm{~Pb}+{ }^{27} \mathrm{Al}$ at $500 A \mathrm{MeV}$ (solid triangles).

As can be observed in Fig. 4(b), the width of the charge distribution of the fission fragments increases when increasing the atomic number of the emitted light charged particle, indicating then an increase of excitation energy. This increase of excitation energy is also predicted by the intranuclear cascade code INCL4.6 [54] (details about this code are given below), as shown in the inset of the figure for the three energies investigated in this work. Finally, the widths of the charge distributions of the fission fragments are very similar for different values of the atomic number of light charged particle. Thus, these results allow us to conclude that this observable characterizes the compound fissioning system independently of the target and/or projectile kinetic energy used for the reaction.

The high excitation energies and low angular momentum of the fissioning nuclei investigated in this work fulfill the optimum conditions for the investigation of dissipative and transient effects in fission [12,15,28,51,61]. Moreover, the combination of light charged particles with the charge distribution of the fission fragments helps us to constrain model calculations, which can be used to investigate the onset of dissipative and transient effects. For this investigation, the code ABLA07 [55] was also modified in order to calculate fission according to the Kramers approach [4], which corresponds to the stationary solution of the fission-decay width.

The ABLA07 code describes the deexcitation of a nucleus emitting $\gamma$ rays, neutrons, light charged particles, and intermediate-mass fragments (IMFs) according to Weisskopf's formalism [62]. For a more realistic description of the deexcitation, the emission barriers for charged particles are also considered by using the Bass potential [63]. These model calculations have been benchmarked in several works by using evaporation residues produced in spallation and fragmentation reactions of nuclei from iron to uranium [16,54,64-68], providing a satisfactory description. Fission is described as a diffusion process across the fission barrier, where the timedependent fission decay width is obtained from an analytical solution of the Fokker-Planck equation according to Ref. [9]. Moreover, the effects of the initial deformation of the fissioning system in the fission decay width are also considered [55]. Finally, the mass- and atomic-number distributions of the fission fragments are described according to a statistical picture [60], where the width of the atomic-number distribution of the fission fragments can be parametrized as a function of the temperature at the saddle-point deformation $\left(T_{s d}\right)$ as

$$
\sigma_{Z}^{2}=\frac{Z_{\mathrm{fiss}}^{2} T_{s d}}{16 d^{2} V / d \nu^{2}},
$$

where $d^{2} V / d v^{2}$ is the second derivative of the potential with respect to the mass-asymmetry degree of freedom at the saddle-point deformation $v=\left(4 / A_{\text {fiss }}\right) /\left(A-A_{\text {fiss }} / 2\right)$, which is obtained from the systematics of the width of mass distributions measured in Ref. [69]. $A_{\text {fiss }}$ and $Z_{\text {fiss }}$ are the mass and atomic numbers of the fissioning nucleus, respectively, and $A$ represents the mass number of the corresponding fragment.

This deexcitation code is coupled to the intranuclear cascade model INCL4.6 [54]. This model provides a complete description of the cascade process, where the characteristics of the remnant (mass, charge, momentum, excitation energy, and intrinsic angular momentum) are derived from the application of conservation laws. The latest version of this model includes, among other things, isospin- and energy-dependent nucleon potentials, an isospin-dependent pion potential, and a new dynamical coalescence algorithm for the production of light clusters (up to masses of $A=8$ ) [54,67]. The predictive power of this code has been validated by using no fissile nuclei $[66,68,70]$.

In Figs. 5(a) and 5(b) we compare the average values of the multiplicity distributions of Fig. 4(a) as a function of the projectile bombarding energy, and the widths of the charge distributions of the fission fragments produced in the reaction ${ }^{208} \mathrm{~Pb}(500 A \mathrm{MeV})+p$ as a function of the atomic number of the emitted particles with model calculations. These calculations reveal that this observable is sensitive to transient time effects. As can be observed in both figures, dynamical calculations based on the Kramers approach for a value of the reduced dissipation parameter of $\beta=4.5 \times 10^{21} \mathrm{~s}^{-1}$ (longdashed line) overestimate the data, while ABLA07 calculations considering dissipative and transient time effects, for the same value of the reduced dissipation parameter (solid line), provide a good description. On the other hand, ABLA07 calculations assuming values of $\beta=10 \times 10^{21} \mathrm{~s}^{-1}$ (dotted line) and $\beta=20 \times 10^{21} \mathrm{~s}^{-1}$ (dot-dashed line) clearly underestimate both measurements.

It should, however, be noticed that these multiplicities of light charged particles are sensitive to dissipative effects, but this sensitivity does not seem sufficient to constrain the value of the dissipation parameter with high precision. This is expected because most of the light charged particles are emitted during the intranuclear cascade process (around 75\%) [54]. The precision of this observable is, however, sufficient to confirm the results obtained previously for the value of the reduced dissipation parameter at small deformations, $\beta=4.5 \times 10^{21} \mathrm{~s}^{-1}$, which leads to a transient time around $1.2 \times 10^{-21} \mathrm{~s}^{1}[28]$.

Light-charged particles are also used in fusion-fission reactions to investigate dissipation. In this case the particles are emitted in the deexcitation process of the compound nucleus only, enhancing the sensitivity to the value of the dissipation parameter. Lestone used this observable to investigate the fission times of light fissioning systems, concluding that a dynamical picture of fission is needed for a correct description of this observable. A similar investigation was carried out by Fröbrich and Gontchar [33], who studied the dependencies of the dissipation parameter. However, the conclusions could be affected by the use of a single observable as pointed out by Vardaci and collaborators $[29,41]$. These authors make use of 

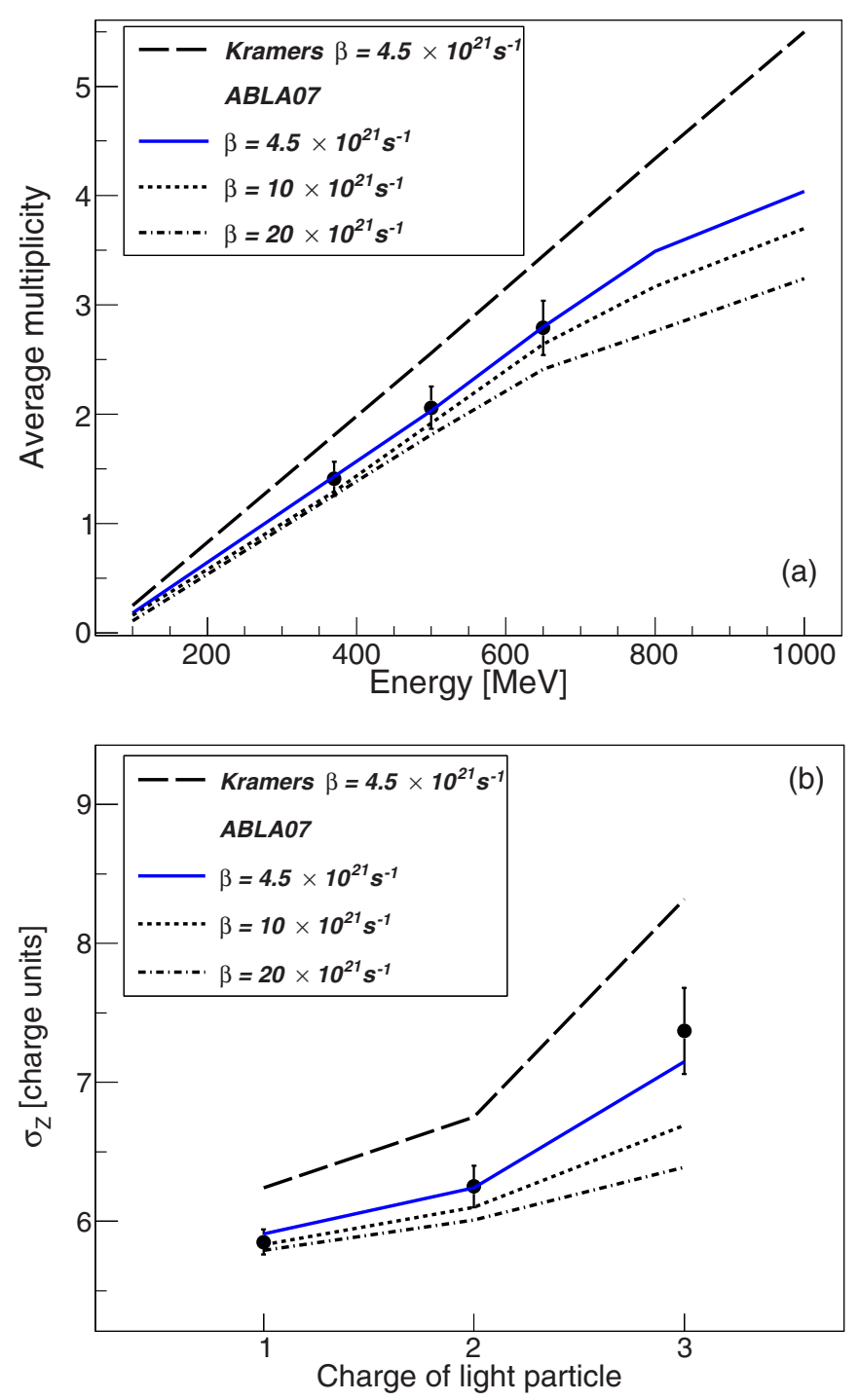

FIG. 5. (a) Average multiplicity of particles with $Z=1$ as a function of the projectile bombarding energy for the reaction ${ }^{208} \mathrm{~Pb}+p$. (b) Width of the atomic-number distribution of the final fission fragments measured in the reaction ${ }^{208} \mathrm{~Pb}(500 \mathrm{~A} \mathrm{MeV})+p$ as a function of the atomic number of the removed light particle from the projectile. In both figures the data are compared with different model calculations (lines).

other observables, such as the evaporation and fission cross sections, to better constrain the ground-to-saddle dissipation parameter by using three-dimensional Langevin calculations. In this case, they also demonstrated that dissipative effects are required to describe the proposed set of observables.

On the other hand, the investigation of light charged particle multiplicities by using spallation reactions shows contradictory results. Lott et al. [36] and Jahnke et al. [35] used direct kinematics to investigate antiproton-induced fission reactions on different nuclei, showing that the data could be consistent with a transient time around $0.5 \times 10^{-21} \mathrm{~s}^{1}$. This conclusion contradicts the results obtained by Tishchenko et al. [37] by using proton-induced fission reactions, pointing out that calculations including dissipative and transient time effects underestimate the fission probability at high excitation energies. However, these conclusions seem to be affected by the model calculations used to reconstruct the excitation energy of the compound systems. As show in Fig. 5(a), the correlation between multiplicity of particles and excitation energy depends on the model calculation. The same correlations can be also found in the interpretation of the particle multiplicities obtained in fusion-fission reactions [1,12,23,27,29,30,42]. This fact could affect the conclusions obtained by Tishchenko et al. [37] because they used a statistical model to obtain the fission probabilities as a function of the excitation energy, and then used this correlation to discard dissipative and transient time effects. On the other hand, at high excitation energies above $500 \mathrm{MeV}(T \sim 6.5 \mathrm{MeV})$, multifragmentation also plays an important role [71,72] that could affect the determination of fission events if the multifragmentation products are counted as fission fragments. This fact could lead to an overestimation of the fission probabilities at the highest excitation energies investigated by Tishchenko et al. [37].

Finally, the results obtained in this work with respect to the dependence on temperature of the dissipation parameter and its magnitude are consistent with the conclusions obtained in most of the works related to the investigation of fission $[1,8,10-14,17,19,20,23,27,29,30,61]$, showing that dissipative and transient time effects are needed to explain the measured multiplicities of light charged particles.

\section{SUMMARY AND CONCLUSIONS}

We have investigated proton-induced fission of ${ }^{208} \mathrm{~Pb}$ in inverse kinematics at different bombarding energies and the reaction ${ }^{208} \mathrm{~Pb}+{ }^{27} \mathrm{Al}$ at $500 \mathrm{~A} \mathrm{MeV}$. We used a highly efficient detection setup that permitted us to identify the atomic number of the fission fragments in coincidence with the light charged particles. With these measurements we were able, for the first time, to determine correlations between the emitted light charged particles and the width of the charge distribution of the fission fragments.

The measurement of the light charged particles in coincidence with the fission fragments has been used for the investigation of dissipative and transient time effects by constraining the initial excitation energy. The comparison of the data with different model calculations shows that the average multiplicity of the emitted particles with $Z=1$ is sensitive to dissipative effects. Moreover, these data also permitted us to study the evolution of the width of the charge distribution of the fission fragments as a function of the atomic number of the particle emitted from the projectile for different entrance channels and bombarding energies. This comparison shows that the width of the charge distribution of the fission fragments depends on the charge of the emitted particle. However, the width depends on neither the entrance channel nor on the bombarding energy. In addition, the comparison with different model calculations indicates that this observable is also sensitive to dissipative effects.

Finally, the overall good description of the light charged particles multiplicities, together with the results showed in our previous works $[28,50,51]$, allowed us to confirm the conclusions obtained with respect to the magnitude and temperature 
independence of the reduced dissipation parameter at small deformations $[10,12-15,18,19,28,51]$, leading to a constant value of $\beta=4.5 \times 10^{21} \mathrm{~s}^{-1}$.

\section{ACKNOWLEDGMENTS}

The authors are grateful to the GSI accelerator staff for providing an intense and stable beam of ${ }^{208} \mathrm{~Pb}$. We also gratefully acknowledge J.-M. Gheller and S. Leray for providing the liquid-hydrogen target and N. Kurz and A. Prochazka for their technical support during the experiment. This work was partially supported by the European Commission under Projects No. ANDES-FP7-249671 and No. CHANDA-FP7605203, the Spanish Ministry of Economy and Competitiveness under Projects No. FPA2010-22174-C02, No. FPA201347831-C2-1-P, and No. Consolider-CPAN-CSD2007-00042, and the Regional Government of Galicia under the program "Grupos de Referencia Competitiva 2013-011". One of us, C.R.T., also acknowledges the support of the Spanish Ministry of Education under a grant of postdoctoral mobility (2011) administered by FECYT.
[1] D. Hilscher and H. Rossner, Ann. Phys. Fr. (Paris) 17, 471 (1992).

[2] D. Jacquet and M. Morjean, Prog. Part. Nucl. Phys. 63, 155 (2009).

[3] N. Bohr and J. A. Wheeler, Phys. Rev. 56, 426 (1939).

[4] H. A. Kramers, Physica 7, 284 (1940).

[5] P. Grangé, L. Jun-Qing, and H. A. Weidenmüller, Phys. Rev. C 27, 2063 (1983).

[6] K. H. Bhatt, P. Grangé, and B. Hiller, Phys. Rev. C 33, 954 (1986).

[7] L. G. Moretto, K. X. Jing, R. Gatti, G. J. Wozniak, and R. P. Schmitt, Phys. Rev. Lett. 75, 4186 (1995).

[8] K. X. Jing, L. W. Phair, L. G. Moretto, Th. Rubehn, L. Beaulieu, T. S. Fan, and G. J. Wozniak, Phys. Lett. B 518, 221 (2001).

[9] B. Jurado, K.-H. Schmidt, and J. Benlliure, Phys. Lett. B 553, 186 (2003)

[10] B. Jurado, C. Schmitt, K.-H. Schmidt, J. Benlliure, T. Enqvist, A. R. Junghans, A. Kelić, and F. Rejmund, Phys. Rev. Lett. 93, 072501 (2004).

[11] J. Benlliure, E. Casarejos, J. Pereira, and K.-H. Schmidt, Phys. Rev. C 74, 014609 (2006).

[12] J. P. Lestone and S. G. McCalla, Phys. Rev. C 79, 044611 (2009).

[13] W. Ye and N. Wang, Phys. Rev. C 87, 014610 (2013).

[14] Y. Ayyad, J. Benlliure, E. Casarejos, H. Álvarez-Pol, A. Bacquias, A. Boudard, M. Caamaño, T. Enqvist, V. Föhr, A. Kelić-Heil, K. Kezzar, S. Leray, D. Mancusi, C. Paradela, D. Pérez-Loureiro, R. Pleskač, J. L. Rodriguez-Sanchez, and D. Tarrio, Phys. Rev. C 89, 054610 (2014).

[15] Y. Ayyad, J. Benlliure, J. L. Rodríguez-Sánchez, A. Bacquias, A. Boudard, E. Casarejos, T. Enqvist, M. Fernandez, V. Henzl, V. Henzlova, B. Jurado, A. Kelić-Heil, T. Kurtukian, S. Lukić, P. Nadtochy, D. Perez-Loureiro, R. Pleskač, F. Farget, M. V. Ricciardi, K. H. Schmidt, C. Schmitt, and S. N. Ngoc, Phys. Rev. C 91, 034601 (2015).

[16] J. Benlliure et al., Nucl. Phys. A 700, 469 (2002).

[17] P. N. Nadtochy, G. D. Adeev, and A. V. Karpov, Phys. Rev. C 65, 064615 (2002).

[18] C. Schmitt, P. N. Nadtochy, A. Heinz, B. Jurado, A. Kelic, and K.-H. Schmidt, Phys. Rev. Lett. 99, 042701 (2007).

[19] C. Schmitt, K.-H. Schmidt, A. Kelić, A. Heinz, B. Jurado, and P. N. Nadtochy, Phys. Rev. C 81, 064602 (2010).

[20] A. Gavron, A. Gayer, J. Boissevain, H. C. Britt, T. C. Awes, J. R. Beene, B. Cheynis, D. Drain, R. L. Ferguson, F. E. Obenshain, F. Plasil, G. R. Young, G. A. Petitt, and C. Butler, Phys. Rev. C 35, 579 (1987).
[21] N. P. Shaw, I. Diószegi, I. Mazumdar, A. Buda, C. R. Morton, J. Velkovska, J. R. Beene, D. W. Stracener, R. L. Varner, M. Thoennessen, and P. Paul, Phys. Rev. C 61, 044612 (2000),

[22] I. Diószegi, N. P. Shaw, A. Bracco, F. Camera, S. Tettoni, M. Mattiuzzi, and P. Paul, Phys. Rev. C 63, 014611 (2000).

[23] I. Diószegi, N. P. Shaw, I. Mazumdar, A. Hatzikoutelis, and P. Paul, Phys. Rev. C 61, 024613 (2000).

[24] J. P. Lestone, Phys. Rev. Lett. 70, 2245 (1993).

[25] M. Thoennessen and G. F. Bertsch, Phys. Rev. Lett. 71, 4303 (1993).

[26] D. J. Hofman, B. B. Back, and P. Paul, Phys. Rev. C 51, 2597 (1995).

[27] P. Fröbrich et al., Nucl. Phys. A 556, 281 (1993).

[28] J. L. Rodríguez-Sánchez et al., Phys. Rev. C 90, 064606 (2014).

[29] E. Vardaci, P. N. Nadtochy, A. Di Nitto, A. Brondi, G. La Rana, R. Moro, P. K. Rath, M. Ashaduzzaman, E. M. Kozulin, G. N. Knyazheva, I. M. Itkis, M. Cinausero, G. Prete, D. Fabris, G. Montagnoli, and N. Gelli, Phys. Rev. C 92, 034610 (2015).

[30] D. J. Hinde, D. Hilscher, and H. Rossner, Nucl. Phys. A 502, 497 (1989).

[31] H. Ikezoe, N. Shikazono, Y. Nagame, Y. Sugiyama, Y. Tomita, K. Ideno, I. Nishinaka, B. J. Qi, H. J. Kim, A. Iwamoto, and T. Ohtsuki, Phys. Rev. C 46, 1922 (1992).

[32] J. P. Lestone, J. R. Leigh, J. O. Newton, D. J. Hinde, J. X. Wei, J. X. Chen, S. Elfstrom, and D. G. Popescu, Phys. Rev. Lett. 67, 1078 (1991).

[33] P. Fröbrich and I. I. Gontchar, Phys. Rep. 292, 131 (1998).

[34] E. Goldenbaum et al., Phys. Rev. Lett. 77, 1230 (1996).

[35] U. Jahnke, W. Bohne, T. von Egidy, P. Figuera, J. Galin, F. Goldenbaum, D. Hilscher, J. Jastrzebski, B. Lott, M. Morjean, G. Pausch, A. Péghaire, L. Pienkowski, D. Polster, S. Proschitzki, B. Quednau, H. Rossner, S. Schmid, and W. Schmid, Phys. Rev. Lett. 83, 4959 (1999).

[36] B. Lott, F. Goldenbaum, A. Bohm, W. Bohne, T. von Egidy, P. Figuera, J. Galin, D. Hilscher, U. Jahnke, J. Jastrzebski, M. Morjean, G. Pausch, A. Péghaire, L. Pienkowski, D. Polster, S. Proschitzki, B. Quednau, H. Rossner, S. Schmid, and W. Schmid, Phys. Rev. C 63, 034616 (2001).

[37] V. Tishchenko, C.-M. Herbach, D. Hilscher, U. Jahnke, J. Galin, F. Goldenbaum, A. Letourneau, and W.-U. Schröder, Phys. Rev. Lett. 95, 162701 (2005).

[38] C.-M. Herbach et al., Nucl. Instrum. Methods Phys. Res., Sect. A 508, 315 (2003).

[39] R. A. Cecil, B. D. Anderson, and R. Madey, Nucl. Instrum. Methods 161, 439 (1979). 
[40] G. Chaudhuri and S. Pal, Phys. Rev. C 65, 054612 (2002).

[41] E. Vardaci et al., Eur. Phys. J. A 43, 127 (2010).

[42] W. Ye, Phys. Rev. C 81, 054609 (2010).

[43] G. Boutoux et al., Phys. Procedia 47, 166 (2013).

[44] T. Gorbinet et al., Phys. Procedia 64, 101 (2015).

[45] E. Pellereau et al., EPJ Web Conf. 62, 06005 (2013).

[46] J. L. Rodríguez-Sánchez et al., EPJ Web Conf. 62, 07009 (2013).

[47] J. L. Rodríguez-Sánchez et al., Phys. Procedia 64, 157 (2015).

[48] J. F. Martin et al., Eur. Phys. J. A 51, 174 (2015).

[49] A. Chatillon et al., EPJ Web Conf. 111, 08001 (2016).

[50] J. L. Rodríguez-Sánchez et al., Phys. Rev. C 91, 064616 (2015).

[51] J. L. Rodríguez-Sánchez et al., Phys. Rev. C 92, 044612 (2015).

[52] B. Voss, in Proceedings of the Nuclear Science Symposium and Medical Imaging Conference (NSS/MIC-IEEE, Valencia, Spain, 2011).

[53] http://geant4.cern.ch/

[54] A. Boudard, J. Cugnon, J.-C. David, S. Leray, and D. Mancusi, Phys. Rev. C 87, 014606 (2013).

[55] A. Kelić, M. V. Ricciardi, and K.-H. Schmidt, in Proceedings of Joint ICTP-IAEA Advanced Workshop on Model Codes for Spallation Reactions, ICTP Trieste, Italy, 4-8 February 2008, edited by D. Filges, S. Leray, Y. Yariv, A. Mengoni, A. Stanculescu, and G. Mank, INDC(NDS)-530 (IAEA, Vienna, 2008), pp. 181-221.

[56] M. F. Rivet, D. Logan, J. M. Alexander, D. Guerreau, E. Duek, M. S. Zisman, and M. Kaplan, Phys. Rev. C 25, 2430 (1982).

[57] M. Gonin, L. Cooke, K. Hagel, Y. Lou, J. B. Natowitz, R. P. Schmitt, S. Shlomo, B. Srivastava, W. Turmel, H. Utsunomiya, R. Wada, G. Nardelli, G. Nebbia, G. Viesti, R. Zanon, B. Fornal, G. Prete, K. Niita, S. Hannuschke, P. Gonthier, and B. Wilkins, Phys. Rev. C 42, 2125 (1990).
[58] A. Chatterjee, A. Navin, S. Kailas, P. Singh, D. C. Biswas, A. Karnik, and S. S. Kapoor, Phys. Rev. C 52, 3167 (1995).

[59] J. Benlliure, A. Grewe, M. de Jong, K.-H. Schmidt, and S. Zhdanov, Nucl. Phys. A 628, 458 (1998).

[60] M. G. Itkis, V. N. Okolovich, A. Ya. Rusanov, and G. N. Smirenkin, Sov. J. Part. Nucl. 19, 301 (1988).

[61] B. Jurado et al., Nucl. Phys. A 747, 14 (2005).

[62] V. F. Weisskopf and D. H. Ewing, Phys. Rev. 57, 472 (1940).

[63] R. Bass, in Proceedings of the Symposium on Deep-Inelastic and Fusion Reactions with Heavy Ions, Berlin 1979 (SpringerVerlag, Berlin, 1980).

[64] A. Boudard, J. Cugnon, S. Leray, and C. Volant, Phys. Rev. C 66, 044615 (2002).

[65] M. V. Ricciardi, P. Armbruster, J. Benlliure, M. Bernas, A. Boudard, S. Czajkowski, T. Enqvist, A. Kelić, S. Leray, R. Legrain, B. Mustapha, J. Pereira, F. Rejmund, K.-H. Schmidt, C. Stephan, L. Tassan-Got, C. Volant, and O. Yordanov, Phys. Rev. C 73, 014607 (2006).

[66] C. Villagrasa-Canton et al., Phys. Rev. C 75, 044603 (2007).

[67] D. Mancusi, A. Boudard, J. Cugnon, J.-C. David, P. Kaitaniemi, and S. Leray, Phys. Rev. C 90, 054602 (2014).

[68] J. Alcántara-Núñez et al., Phys. Rev. C 92, 024607 (2015).

[69] A. Ya. Rusanov, M. G. Itkis, and V. N. Okolovic, Phys. At. Nucl. 60, 683 (1997).

[70] D. Mancusi, A. Boudard, J. Cugnon, J.-C. David, T. Gorbinet, and S. Leray, Phys. Rev. C 84, 064615 (2011).

[71] K.-H. Schmidt et al., Nucl. Phys. A 710, 157 (2002).

[72] J. B. Natowitz, R. Wada, K. Hagel, T. Keutgen, M. Murray, A. Makeev, L. Qin, P. Smith, and C. Hamilton, Phys. Rev. C 65, 034618 (2002). 